\title{
Vertebrobasilar ischemia (VBI) related neuro-ophthalmic syndromes after routine activities involving hyperextension or rotation of the neck (hairdresser syndrome)
}

\author{
Jonathan A. Go ${ }^{1} \cdot$ Bayan Al Othman ${ }^{2} \cdot$ Ashwini Kini $^{2} \cdot$ Hilary A. Beaver ${ }^{2} \cdot$ Andrew G. Lee $^{1,2,3,4,5,6,7}$
}

Received: 23 April 2019 / Accepted: 15 September 2019 / Published online: 30 September 2019

(c) The Author(s), under exclusive licence to The Royal College of Ophthalmologists 2019

\begin{abstract}
Background Vertebrobasilar insufficiency (VBI) after rotation or hyperextension of the neck during otherwise routine activities is uncommon "hairdresser syndrome" (HDS). We report three such cases presenting with neuro-ophthalmic complaints (Horner syndrome, ophthalmoplegia, and transient vision loss).

Methods A retrospective review was performed of the electronic health records of three patients seen in the neuroophthalmology clinic of Houston Methodist Hospital with acute neuro-ophthalmological signs after neck hyperextension. A literature review was also performed.

Results While various ischemic signs of VBI have been previously documented, to our knowledge, these are the first cases in the English ophthalmic literature to describe the presenting neuro-ophthalmologic signs of HDS.

Conclusion Clinicians should consider the possibility of neck hyperextension as a potential mechanism and precipitating event for acute neuro-ophthalmic presentations of VBI. Patients with a history of occupational (e.g., carpenter), recreational (e.g., bow hunter), or cosmetic (hairdresser) neck hyperextension (i.e., possible HDS) should undergo structural and vascular neuroimaging of both the head and neck (e.g., CT/CTA or MRI/MRA) to look for evidence of posterior fossa ischemia and/ or vertebrobasilar lesion (e.g., occlusion, dissection, FMD, and pseudoaneurysm).
\end{abstract}

\section{Introduction}

Vertebrobasilar insufficiency (VBI) after rotation or hyperextension of the neck during otherwise routine

Andrew G. Lee

aglee@houstonmethodist.org

1 Baylor College of Medicine, 1 Baylor Plaza, Houston, TX 77030, USA

2 Department of Ophthalmology, Blanton Eye Institute, Houston Methodist Hospital, 6550 Fannin St, Houston, TX 77030, USA

3 Departments of Ophthalmology, Neurology, and Neurosurgery, Weill Cornell Medicine, 1305 York Ave, New York, NY 10021, USA

4 Department of Ophthalmology, University of Texas Medical Branch, 700 University Blvd, Galveston, TX 77555, USA

5 University of Texas MD Anderson Cancer Center, 1515 Holcombe Blvd, Houston, TX 77030, USA

6 Texas A and M College of Medicine, 8447 Bryan Rd, Bryan, TX 77807, USA

7 Department of Ophthalmology, The University of Iowa Hospitals and Clinics, 200 Hawkins Drive, Iowa City, IA 52242, USA activities (e.g., shampoo and hair wash at the hairdresser, recreational, or occupational related head/neck positioning) is uncommon "hairdresser syndrome" (HDS). We report three such cases presenting with neuro-ophthalmic complaints (Horner syndrome, ophthalmoplegia, and transient vision loss). While various ischemic signs of VBI have been previously documented, to our knowledge, these are the first cases in the English ophthalmic literature to describe the presenting neuro-ophthalmologic signs of HDS. Clinicians should consider the possibility of traumatic neck hyperextension-related vertebrobasilar ischemia in patients presenting with acute neuro-ophthalmic afferent and/or efferent symptoms and signs (e.g., visual loss, diplopia, anisocoria, and ptosis) after apparently routine activities.

\section{Methods}

A retrospective review was performed of the electronic health records of three patients seen in the neuroophthalmology clinic of Houston Methodist Hospital with acute neuro-ophthalmological signs after neck 
Fig. 1 a In case number one, external photography revealed a $2 \mathrm{~mm}$ ptosis OS and anisocoria (with a smaller left pupil) more prominent in the dark. b MRA of the neck without contrast revealed mild luminal irregularity in the midcervical, internal carotid arteries bilaterally, possibly indicating fibromuscular dysplasia. A possible $3 \mathrm{~mm}$ pseudoaneurysm was noted on the right (white arrow)
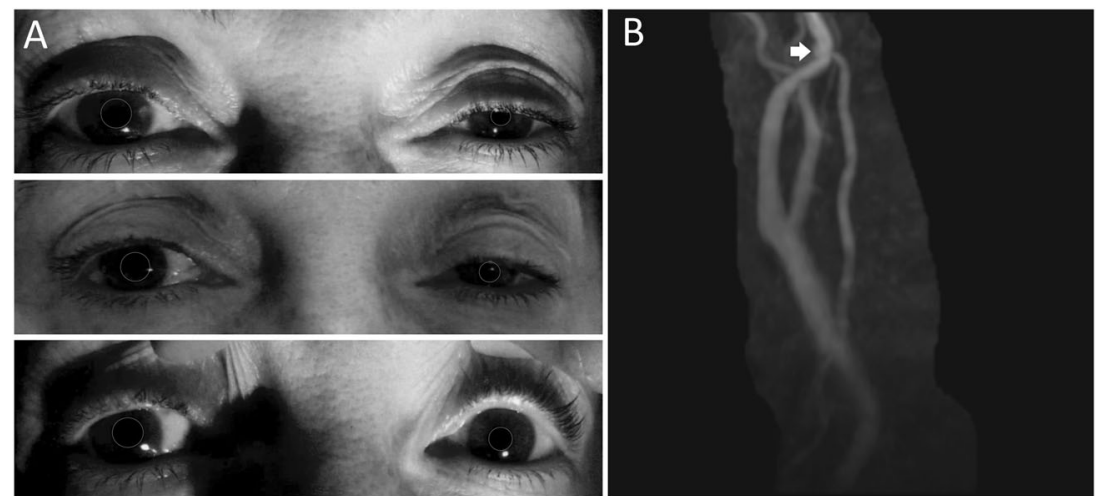

hyperextension. Data collected included the presenting complaint, past medical history, neuroimaging, operative and postoperative records, and neuro-ophthalmology consultation results. A literature review was also performed using combinations of the search terms: "Hairdresser syndrome", "Beauty parlor syndrome", "Horner syndrome", "ophthalmoplegia", and "vision loss". Patient consent was received for both medical photography and case publication. Potential personal identifying health information was excluded. This study was approved by the Institutional Review Board (IRB) of Houston Methodist Hospital.

\section{Case one}

A 74-year-old woman presented with a 2-day history of new onset left (OS) ptosis. Her past medical history was significant for nonspecific anxiety and prior cosmetic botulism toxin injections last performed 4 months earlier. Her past surgical history, past ocular history were unremarkable, and her review of systems was unremarkable.

The patient noticed her left eyelid becoming "swollen, itchy, and heavy" while she was receiving a hair wash (in the sitting position with posterior neck hyperextension) at the hairdresser salon. When she returned home that same day, she noticed significant dropping of her left eyelid. She consulted her dermatologist, who recommended an emergency department evaluation and subsequently referred for neuro-ophthalmology evaluation.

Neuro-ophthalmic examination revealed best corrected visual acuity (BCVA) of 20/20 in both eyes (OU). Visual fields were full OU. The patient's right pupil measured 5 $\mathrm{mm}$ in the dark and $3 \mathrm{~mm}$ in the light, and the left pupil measured $4 \mathrm{~mm}$ in the dark and $2.5 \mathrm{~mm}$ in the light. No relative afferent pupillary defect (RAPD) was noted OU. A $2 \mathrm{~mm}$ upper eyelid ptosis and a $1 \mathrm{~mm}$ inverse ptosis was noted OS (Fig. 1a), and dilation of the left pupil occurred following instillation of $0.5 \%$ apraclonidine eye drop OU, consistent with a pharmacologically proven left Horner syndrome. Anterior segment, intraocular pressure, extraocular motility, and dilated fundus exam were unremarkable OU.

Magnetic resonance (MR) imaging of the brain without contrast was normal. MR angiography (MRA) of the neck revealed luminal irregularity in the midcervical, internal carotid arteries consistent with fibromuscular dysplasia (FMD) bilaterally, and a wall abnormality suggestive of a 3 $\mathrm{mm}$ pseudoaneurysm on the left. No other significant abnormalities, stenosis, or dissection was noted in the common carotid artery bifurcations and cervical vertebral arteries (Fig. 1b). Standard catheter angiography showed only FMD changes and no aneurysm or pseudoaneurysm. The patient was started on low dose aspirin daily and remained stable at last follow up visit.

\section{Case two}

An 82-year-old woman presented with acute onset of ataxia and painless blurry vision 9 months prior to neuro-ophthalmic evaluation. Her past medical history was significant for hypertension, hyperlipidaemia, and atherosclerotic carotid artery disease. Her past surgical history was significant for tonsillectomy and a carotid stent placement. Past ocular history was significant for dry eyes, primary open angle glaucoma OU, cataract surgery OU, and a retinal detachment OS requiring retinal laser therapy, air fluid gas exchange, and scleral buckle OS. She had a prior macular hole and proliferative vitreoretinopathy OS that was stable at counting fingers vision OS. Her medications prior to presentation were losartan, rosuvastatin, vitamin $\mathrm{D}$, latanoprost, and timolol eye drops. Her review of systems was unremarkable.

The patient reported acute neurologic symptoms after having her hair washed in a sitting position with her the neck hyperextended at the beauty parlor. She noticed she "could not walk straight", had binocular diplopia, and had to "bend over" to walk. She returned home and her symptoms improved but she was left with mild residual symptoms. The patient was seen on the neuro-ophthalmology service several months later. 
Neuro-ophthalmic examination revealed BCVA of 20/25 OD and count fingers OS (due to prior retinal detachment OS). A RAPD was present OS. Motility examination revealed a 30-prism dioptre exotropia (XT), with an adduction lag OD when looking to the right, consistent with a possible right internuclear ophthalmoplegia. No saccadic abnormality or nystagmus was noted. There was mechanically limited elevation and a mild abduction deficit noted OS presumed to be at least partially secondary to the prior retinal buckle OS. Intraocular pressure measurements were $13 \mathrm{~mm}$ $\mathrm{Hg}$ OD and $7 \mathrm{~mm} \mathrm{Hg}$ OS. Mechanical $1 \mathrm{~mm}$ of lid retraction OS was noted secondary to the scleral buckle effect superiorly OS. Fundoscopic examination showed diffuse optic atrophy OS and healthy-looking optic disc OD. Diffuse stable and inactive peripheral chorioretinal scarring was noted OS from the prior retinal detachment and surgery.

MRI of the brain with and without contrast (Fig. 2a, b) showed an infarct in the right cerebellum and brainstem consistent with prior vertebrobasilar ischemia but no acute ischemic change, haemorrhage, or mass effect. MRI of the orbits with and without contrast revealed no focal mass. MRA of the head without contrast revealed no aneurysm or hemodynamically significant stenosis of the intracranial arterial circulation. MRA of the neck without contrast (Fig. 2c) was unremarkable, with no significant stenosis or dissection. Patency was noted in the carotid arteries and vertebral arteries bilaterally. The right vertebral artery was hypoplastic but grossly patent. A full stroke evaluation was negative, and the patient was treated with aspirin. The patient remained stable at last follow up.

\section{Case three}

A 63-year-old man presented with 2 years of recurrent, painless, transient, bilateral, and simultaneous vision loss. He described these episodes as severe and acute, with no light perception vision OU for several minutes with no loss of consciousness. His past medical history was significant for well controlled essential hypertension, abdominal aortic aneurysm, type 2 diabetes mellitus with no diabetic retinopathy, hyperlipidaemia, and cervical degenerative disk disease. His past surgical history was significant for carpal tunnel surgery and a plate and screw placement at C6-C7 for his cervical degenerative disk disease. Two years earlier, he was involved in a traumatic motor vehicle accident caused whiplash and displacement of his installed plate, requiring C5-C6-C7 fusion surgery for upper left extremity paraesthesia. He continued to suffer from paraesthesia post surgery and received a steroid injection at C6-C7. Two days post injection, he reported his first episode of transient vision loss. The patient worked as a carpenter and reserve police officer.

The patient's episodes were usually precipitated by neck position, but especially neck rotation and neck hyperextension. He was sometimes required to work in the supine position during his occupation as a carpenter and would have to assume awkward positions involving neck hyperextension on the job. He sometimes also noted the same symptoms after neck rotation when looking over his shoulder while backing or parking his car. He was asymptomatic between episodes.

Neuro-ophthalmic examination revealed BCVA of 20/20 OU. Humphrey visual fields showed nonspecific superior arcuate defect nerve fibre layer defects OU. Pupils measured $4 \mathrm{~mm}$ in the dark and $2 \mathrm{~mm}$ in the light with no RAPD OU. Anterior segment exam, intraocular pressure, dilated fundus exam, and extraocular motility were unremarkable.

MRI and MRA of the head and neck with and without contrast showed no evidence of vertebral artery dissection or stenosis. There was no spinal misalignment, no plate migration, no complications from steroid injection, and normal spinal cord diameter. Doppler ultrasound (U/S) revealed no significant carotid stenosis bilaterally. Vertebral artery head turning manoeuvre did not show significant velocity change, and flow direction was anterograde in all
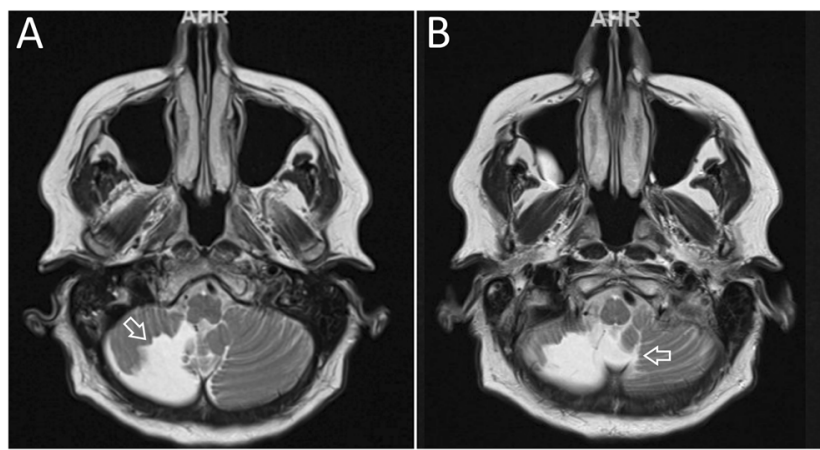

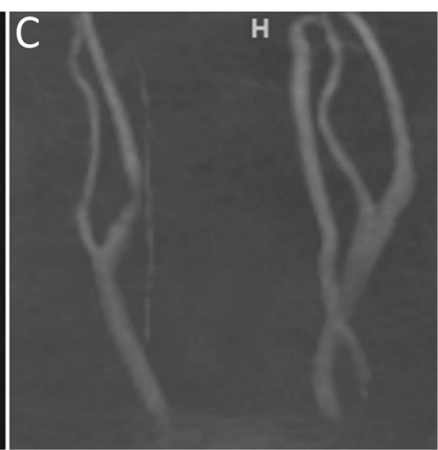

indicating asymmetric atrophy. $\mathbf{c}$ MRA of the neck was unremarkable, with no significant stenosis or dissection. The right vertebral artery was hypoplastic but grossly patent
Fig. 2 a In case number two, MRI of the brain showed a chronic insult in the right cerebellum. b MRI of the orbits revealed no focal mass. Asymmetric T2 signal was present in the left optic nerve, likely 
head positions. The left vertebral artery appeared to be dominant, and the right vertebral artery terminated in the right posterior inferior cerebellar artery, effectively creating a hypoplastic segment. Computed tomography (CT) scan of the head with and without contrast showed chronic, opacified, inflammatory changes in the left frontal sinus, and no acute intracranial abnormalities. Computed tomography angiography (CTA) of the head with and without contrast showed no dissection, stenosis, or aneurysm in the major branches of anterior and posterior circulation. He was prescribed low dose aspirin daily, and he was advised to control his vasculopathic risk factors and to avoid further hyperextension and hyperrotation of the neck.

\section{Discussion}

Neck hyperextension or rotation can affect structure and flow of the vertebral arteries (e.g., occlusion, dissection, or pseudoaneurysm). Neck rotation in the "bow hunter syndrome" occurs from the rotation inherent in appropriate archery-related aiming and firing positions. Unfortunately, the neck rotation and neck hyperextension can precipitate VBI or in rare cases stroke [1-3]. Likewise, the "hairdresser syndrome" or "beauty parlor syndrome" is a cerebrovascular event following neck hyperextension in the sitting position that is a common commercial hair washing/ shampoo position [4, 5]. However, recent studies suggest dissection from abnormal neck stretching and aggravation of previous atherosclerotic disease may be more likely associated with HDS [6] rather than vertebral artery impingement [7]. In addition to classic stroke symptoms, other neurological signs such as cervical radiculopathy [8] and vertigo [9] have been reported from HDS. However, to our knowledge, these three cases represent the first report of neuro-ophthalmological findings of VBI after routine activities with neck position (i.e., HDS) without evidence of occlusion $[10,11]$ to be reported in the English language ophthalmic literature.

Neck hyperextension and neck rotation are common in everyday activities and do not typically produce symptoms in otherwise healthy individuals. Predisposing risk factors however may increase the risk of HDS. In case number one, the presence of FMD could have contributed to the HDS. FMD is a nonatherosclerotic, noninflammatory vascular disorder associated with cervicocranial artery dissection [12], classically diagnosed in young, premenopausal women. However, in actuality, its reported mean diagnosis age is 51.9 years [13]. Horner syndrome is rarely associated with FMD, with a $4.7 \%$ reported occurrence in the US Registry for FMD [13]. The presentation of Horner syndrome and FMD is frequently accompanied by carotid dissection (CD) [5, 14, 15]. While our patient's MRA did suggest FMD, there was no evidence of any acute dissection but healed dissection cannot be excluded. To our knowledge, there is only one other documented case of Horner syndrome and FMD in English ophthalmic literature that explicitly reports a lack of CD [13]. In case number two, there was likely multifactorial contribution from both chronic, residual retinal injury OS, and acute posterior fossa ischemic injury from neck hyperextension (i.e., right INO presumed due to a cerebellar/brainstem infarct). The hypoplastic right vertebral artery may have increased the probability that any left-sided insult, even physiological, could precipitate an ischemic event. In case number three the patient had multiple, stereotyped episodes of bilateral simultaneous no light perception vision during neck hyperextension suggesting vertebrobasilar ischemia,

Clinicians should consider the possibility of neck hyperextension as a potential mechanism and precipitating event for acute neuro-ophthalmic presentations of VBI. Patients with a history of occupational (e.g., carpenter), recreational (e.g., bow hunter), or cosmetic (hairdresser) neck hyperextension (i.e., possible HDS) should undergo structural and vascular neuroimaging of both the head and neck (e.g., CT/CTA or MRI/MRA) to look for evidence of posterior fossa ischemia and/or vertebrobasilar lesion (e.g., occlusion, dissection, FMD, and pseudoaneurysm).

\section{Summary}

\section{What was known before}

- Vertebrobasilar insufficiency (VBI) can occur after neck rotation or hyperextension.

- Associated activities include shampoo and hair wash at the hairdresser and recreational or occupational head positioning.

- This phenomenon is known as "hairdresser syndrome" (HDS).

\section{What this study adds}

- To our knowledge, these are the first cases in the English ophthalmic literature to describe the presenting neuroophthalmologic signs of HDS.

- Signs include Horner syndrome, ophthalmoplegia, and transient vision loss.

- Clinicians should consider the possibility of traumatic, neck hyperextension-related VBI in patients presenting with acute neuro-ophthalmic afferent and/or efferent symptoms and signs. 
- Patients should undergo structural and vascular neuroimaging of both the head and neck (e.g., CT/CTA or MRI/MRA).

Acknowledgements This research did not receive any specific grant from funding agencies in the public, commercial, or not-for-profit sectors. There are no relevant financial disclosures for any author. No conflicting relationships exist for any author. All authors attest that they meet the current ICMJE criteria for authorship. This material is original research, has not been previously published, and has not been submitted for publication elsewhere while under consideration.

\section{Compliance with ethical standards}

Conflict of interest The authors declare that they have no conflict of interest.

Publisher's note Springer Nature remains neutral with regard to jurisdictional claims in published maps and institutional affiliations.

\section{References}

1. Brinjikji W, Graffeo CS, Perry A, et al. Moving target: transient rotational stenosis precipitating jugular bow hunter's syndrome. J Neurointerv Surg. 2017;9:e28. https://doi.org/10.1136/neurintsurg2016-012827.rep.

2. Duan G, Xu J, Shi J, Cao Y. Advances in the pathogenesis, diagnosis and treatment of bow hunter's syndrome: a comprehensive review of the literature. Interv Neurol. 2016;5:29-38. https://doi.org/10.1159/000444306.

3. Strickland BA, Pham MH, Bakhsheshian J, Russin JJ, Mack WJ, Acosta FL. Bow Hunter's syndrome: Surgical management (video) and review of the literature. World Neurosurg. 2017;103:953.e7-.e12. https://doi.org/10.1016/j.wneu.2017.04. 101.
4. Hennings JM, Hoehn D, Schumann-Spaeth E, Weber F. Painless hypoglossal palsy as an isolated symptom of spontaneous carotid dissection. J Stroke Cerebrovasc Dis. 2014;23:1988-90. https:// doi.org/10.1016/j.jstrokecerebrovasdis.2014.02.001.

5. Heckmann JG, Heron P, Kasper B, Dörfler A, Maihöfner C. Hairdressser syndrome. Cerebrovasc Dis. 2006. https://doi.org/10. 1159/000090449.

6. Correia PN, Meyer IA, Eskandari A, Michel P. Hairdresser syndrome revisited: an 11-year single-center consecutive series. Int J Stroke. 2016. https://doi.org/10.1177/1747493015620809.

7. Foye PM, Najar MP, Camme A, et al. Prospective study of pain, dizziness, and central nervous system blood flow in cervical extension: vascular correlations to hairdresser syndrome and salon sink radiculopathy. Am J Phys Med Rehabil. 2002. https://doi.org/ 10.1097/00002060-200206000-00001.

8. Stitik TP, Nadler SF, Foye PM. Salon sink radiculopathy: a case series. Am J Phys Med Rehabil. 1999. https://doi.org/10.1097/ 00002060-199907000-00019.

9. Endo K, Ichimaru K, Shimura H, Imakiire A. Cervical vertigo after hair shampoo treatment at a hairdressing salon: a case report. Spine 2000;25:632-4. https://doi.org/10.1097/00007632-20000301000017.

10. Moncayo J, Bogousslavsky J. Vertebro-basilar syndromes causing oculo-motor disorders. Curr Opin Neurol. 2003;16:45-50. https:// doi.org/10.1097/01.wco.0000053591.70044.8c.

11. Devuyst G, Bogousslavsky J, Meuli R, Moncayo J, de Freitas G, van Melle G. Stroke or transient ischemic attacks with basilar artery stenosis or occlusion: clinical patterns and outcome. Arch Neurol. 2002;59:567-73. http://www.ncbi.nlm.nih.gov/pubmed/ 11939891. Accessed 14 July 2019.

12. Buttinelli C, Spalloni A, Fieschi C, Rasura M. Migraine and arterial dissection in a young woman. Neurol Sci. 2001;22:275-8.

13. McInnis CP, Haynor DR, Francis CE. Horner syndrome in fibromuscular dysplasia without carotid dissection. Can J Ophthalmol. 2016;51:e53-5. https://doi.org/10.1016/j.jcjo.2015.10.017.

14. Chan CC, Paine M, O'Day J. Carotid dissection: a common cause of Horner's syndrome. Clin Exp Ophthalmol. 2001;29:411-5.

15. Dziewas R, Konrad C, Dräger B, et al. Cervical artery dissection Clinical features, risk factors, therapy and outcome in 126 patients. $\mathrm{J}$ Neurol. 2003. https://doi.org/10.1007/s00415-003-0174-5. 